\title{
Differential Fluxes of Heat and Salt: IMPLICATIONS FOR CIRCULATION AND ECOSYSTEM MODELING
}

\author{
By Barry Ruddick
}

\section{What Are Differential Fluxes, and Why Might They Matter?}

$\mathrm{T}$ HE SALTS DISSOLVED in the world's oceans have profound effects, from the large scale, where evaporation-precipitation patterns have the opposite buoyancy effect to thermal forcing, to the microscale, where molecular diffusion of heat is 70 times faster than that of salt. It has often been assumed that this difference can only matter at the smallest scales, so that heat and salt are mixed in exactly the same way by turbulence. However, the different molecular diffusivities are the basis for a variety of phenomena known as double-diffusion, and these can lead to important differences between heat and salt fluxes, with consequences for much larger scales. In particular,

Density fluxes the "wrong way": $\mathrm{K}_{\rho}<0$.

Barry Ruddick, Department of Oceanography, Dalhousie University, Halifax, N.S., B3H 4J1 Canada.

* Most of the Central Waters of the subtropical oceans is stratified in this sense, due to surface heating and evaporation.

† This situation may be visualized surprisingly easily by filling a conveniently sized tank with roomtemperature fresh water, dissolving a pinch of flourescein salts in a cup or two of water that is nearly boiling temperature. The hot flourescein mixture is than carefully floated on the top of the fresh water by pouring onto a piece of floating cardboard. As the top layer cools by heat loss to the air, conditions become perfect for the formation of relatively large heat-flourescein fingers, which can be made visible via a slit projector light source. I am indebted to two theoreticians. George Veronis and Oliver Kerr, for pointing this out to me. A movie of this and other laboratory demonstrations can be found $\mathrm{PO}$ demos web page, accessable through the Dalhousie Oceanography home page. $<$ http:www.phys.ocean.dal.ca/Da/Ocean_Home.html>
Conventional turbulent mixing, for which heat, salt, and density diffusivities are equal and positive, reduces contrasts in density. In contrast, double diffusion can increase density contrasts, and this is the key to understanding many of its oceanic consequences: the possibility of mixing without an external source of kinetic energy, formation of regular series of layers, the strange effects on vertical motion and stretching that modulate thermohaline circulation, and even lateral mixing over thousands of kilometers via thermohaline intrusions.

The best-known example of doublediffusion is that of salt fingers, which occur spontaneously when warm, salty water (green in Fig. 1, with the red dots representing heat) overlies cooler, fresher water (blue in Fig. 1).* Consider what happens to a downward finger like perturbation of the interface between the green and blue. The increased surface area offers an opportunity for enhanced heat exchange by molecular diffusion, which cools off the warm, salty water. Since the molecular salt diffusivity is 70 times smaller, the "green finger" becomes more dense, and therefore falls downward. The cooler, fresher blue water receives the heat, becomes less dense, and rises. The net effect is a growing field of nearly vertical fingers in which vertical advection of salt by the fingers is the most important feature. The vertical motion is enabled by the lateral diffusion of heat between the fingers, but requires the density difference of the salt field to drive it. $\dagger$

When we consider what happens in a laboratory tank over time scales of $\sim 1 \mathrm{~h}$, we find that the salt is carried downward by the fingers and the top-to-bottom contrast is reduced to nearly zero- a positive eddy diffusivity for salt. Heat is also carried downward by the fingers, but to a lesser extent because it is short-circuited by lateral diffusion. The heat contrast is also reduced, but not to zero. Note that the potential energy of the salt field is lowered, and that of the temperature field is raised. The surprising thing is discovered when we add up the contributions of heat and salt to the density profiles - the

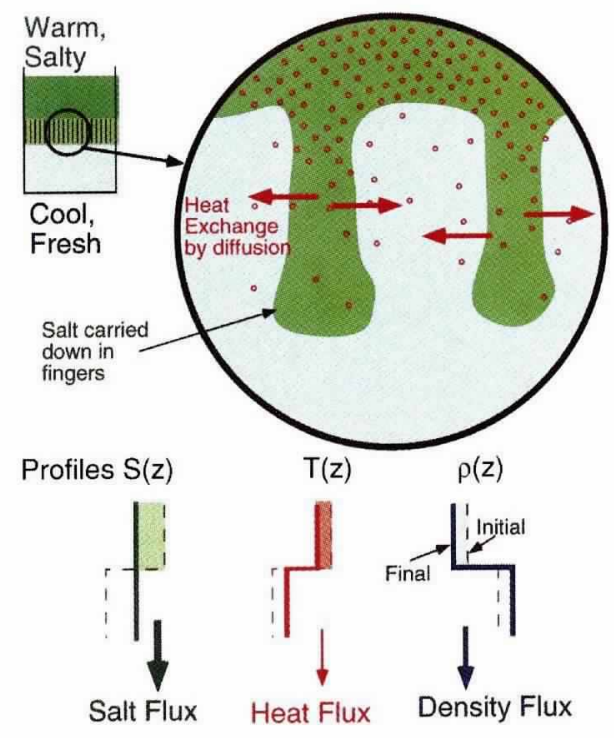

Fig. 1: The mechanism of salt fingers. Finger-like perturbations allow enhanced molecular diffusion of heat (red dots) between warm salty water and cold fresh water. The warm salty water becomes cooler and more dense, and sinks. The converse occurs to the cold fresh water. The macroscale effect of salt fingers is a downward flux of salt in the fingers, and a smaller downward flux of heat. The density flux is also downward, which enhances the density contrast between layers. 
density of the upper layer (which was lighter than the lower one to begin with) decreases, mostly due to loss of salt, and the converse occurs to the lower layer. The flux of density is downward (i.e., up the density gradient), which constitutes the net release of potential energy that drives the salt fingering. The differences in density are actually increased over time-in other words, a negative eddy diffusivity for density!

When warmer saltier water underlies cool, fresh water, as often occurs in subpolar waters, a different form of instability, called diffusive convection, can occur. Consider the motion of a small fluid parcel (simulated by a Cartesian Diver in Fig. 2) $\neq$ in such a field. If the diver is perturbed downward into warmer water, it will gain heat from its surroundings and become lighter. This will make it rise in the density gradient to cooler levels, where it loses the heat, becomes heavier, and falls. The time lag required for thermal diffusion changes an ordinary buoyancy oscillation into an overstable growing oscillation, as was observed by Shirtcliffe (1967). The bobbing fluid parcel acts as a shuttle bus for the heat, transferring it upward, while the small molecular diffusion of salt keeps the salt flux from becoming large. The net result is upward heat and salt fluxes (positive eddy diffusivities) and a downward density flux, again with a negative eddy diffusivity for density. The result of this growing oscillation can be a breakdown of smooth stratification into layers, as described in the next section.

Gargett (1988) has raised the possibility that the different molecular diffusivities of heat and salt can result in different eddy diffusivities for heat and salt in stratified turbulence (hence the term "differential fluxes" in the title of this article). The resulting buoyancy flux and its convergence could lead to many of the phenomena described below, even when $\mathrm{T}$ and $\mathrm{S}$ are stably stratified and there is no direct source of potential energy for mixing. However, not much is known

$\$$ The self-propelled diver is demonstrated on the $\mathrm{PO}$ demos web page, accessable through the Dalhousie Oceanography home page <http://www. phys.ocean.dal.ca/DalOcean_Home.html>.

$\S$ Look for a demonstration of this experiment on the PO demos web page, accessable through the Dalhousie Oceanography home page <http://www. phys.ocean.dal.ca/DalOcean_Home.html>.

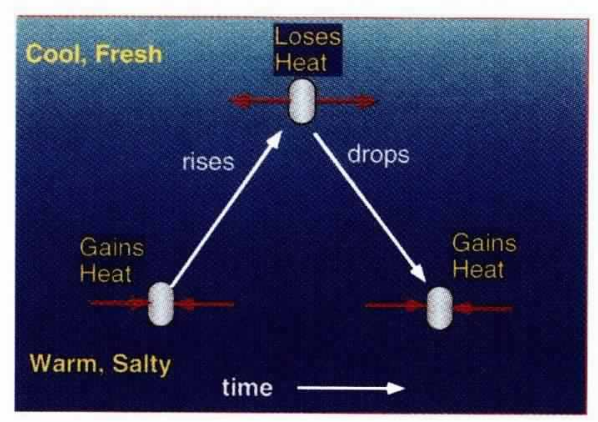

Fig. 2: The self-propelled Cartesian diver.

quantitatively about differential turbulent mixing, and so it won't be discussed further here.

\section{Are Double-Diffusive Fluxes Ever Large Enough to Matter?}

The phenomena of salt fingers and diffusive convection are together known as "double-diffusive convection" (Turner 1973). When double-diffusion was first discovered, the fact that it is a form of "self-driven" turbulence that extracts the potential energy of the salt field (in the case of fingers) or the temperature field (in the case of diffusive convection) caused speculation that double-diffusive fluxes might be quite large. The quiet, efficient, and steady nature of double-diffusion made oceanographers think that it could be the "tortoise" compared with the energetic but intermittent "hare" of internal wave mixing. In this section we examine how double-diffusive fluxes are enhanced by the formation of layers, how laboratory measurements have been successfully verified in the ocean to quantify diffusive fluxes, and how the case seems to be much more complicated for salt fingers.

\section{Layer Formation}

Huppert and Linden (1979) demonstrated one mechanism that causes a smooth salt stratification to break down into a series of well-mixed layers separated by high-gradient "steps." This was important because continuous profiles of $\mathrm{T}$ and $\mathrm{S}$ from the newly invented Conductivity Temperature Depth profiler (CTD) often showed coincident steps and layers in both $\mathrm{T}$ and $\mathrm{S}$ that were dubbed "thermohaline staircases," and Huppert and Linden explained their formation. In their experiment, a stable salinity gradient was subjected to a steady heat flux from below.§ After a convecting layer forms and becomes warmer than the region above, molecular diffusion of heat in the region above the layer creates a gradient region with diffusive sense stratification (warm salty underlying cooler fresher). As the thermal boundary layer grows, the gradient region eventually becomes unstable, either to the overstable oscillations or to Rayleigh convection. The region then breaks down and forms a new convecting layer, and molecular heat diffusion above this layer starts the process anew. The resulting series of layers separated by sharp interfaces is called a diffusive thermocline staircase, often found in subpolar regions of the ocean, and in geothermally heated saline lakes. The heat and salt fluxes across the interfaces are thought to be via molecular diffusion, whereas the fluxes are carried through the layers by convection (Fig. 3).

The downward salt flux by salt fingers can form layers in a similar fashion, by fluxing density into a stable stratification, and reducing the density gradient to the point of convection.

It is possible (although very incomplete) to view layer formation in terms of a negative eddy diffusivity for density. The diffusion equation describes the evolution of the density field, and with a positive diffusivity $\mathrm{K}$, tells us that small perturbations decay with increasing time:

$$
\frac{\partial \rho}{\partial \mathrm{t}}=\frac{\partial}{\partial \mathrm{z}}\left(\mathrm{K} \frac{\partial \rho}{\partial \mathrm{z}}\right)
$$

However, substituting $-K$ for $K$ is the same as substituting $-\mathrm{t}$ for $\mathrm{t}$. The diffusion equation is therefore unstable for negative $\mathrm{K}$, so that perturbations will grow with time. The upgradient fluxes pile up into the perturbations and cause them to grow.

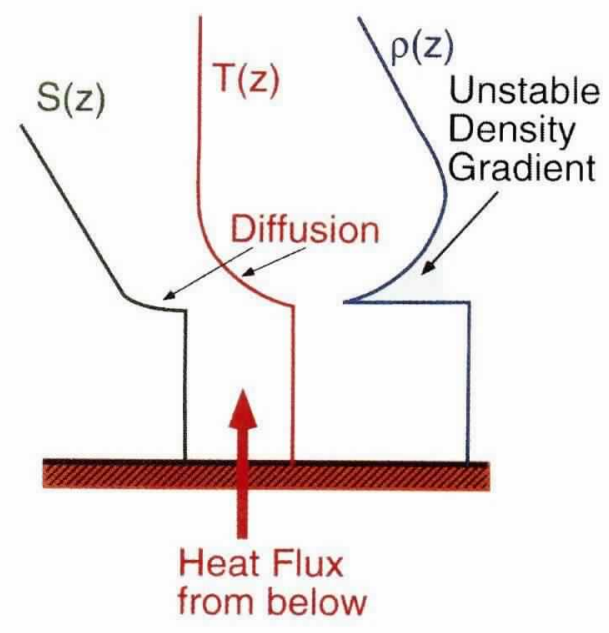

Fig. 3: Formation of layers in a salt gradient by heating from below. 
The main consequence of layer formation is to increase the fluxes over the molecular value, and since thicker layers mean larger steps, then thicker layers mean larger fluxes. Qualitatively we can say that double-diffusion matters most where layers occur, because that is where it is dominant enough to make layers, and because the fluxes are larger with the layers.

Diffusive Convection in Arctic Waters

Padman and Dillon (1987, 1989) measured the temperature microstructure with millimeter resolution in a diffusive thermohaline staircase in the Arctic Canada Basin. They were able to estimate the temperature gradient and so the diffusive heat flux across the interfaces, and found agreement with the extrapolation of the $\Delta T^{4 / 2}$ laboratory flux laws (reviewed by Turner. 1973) to oceanic values. They were also able to estimate the size and lifetime of the convecting thermally anomalous blobs, and found good agreement with observations. Furthermore. the laboratory laws predicted a very small buoyancy flux and hence a rate of turbulent energy dissipation that should lie below instrumental noise levels. This was indeed observed.

Kelley (1984) noted that, if we could predict the thickness of layers, $H$, in a thermohaline staircase, then, given the large-scale gradients, the interfacial jumps in $\mathrm{S}$ and $\mathrm{T}$ would be known, hence we could predict the fluxes. He applied dimensional reasoning to argue that the dimensionless group

$$
\frac{\mathrm{H}}{\sqrt{\kappa_{\mathrm{T}} / \mathrm{N}}}
$$

where $\mathrm{N}$ is the buoyancy frequency and $\kappa_{\mathrm{T}}$ the molecular diffusivity of heat, should be a function of the density ratioll $\mathrm{R}_{p}=\beta \Delta \mathrm{S} / \alpha \Delta \mathrm{T}$ and numbers characterizing fluid properties such as the Prandtl number. He found this to be the case for the observations published before 1984. and used this systematic dependence and the "4/3 flux laws" to propose effective eddy diffusivities for diffusively stratified ocean regions (Fig. 4). The observations made by Padman and Dillon (1987. 1989) from the Canada Basin fit Kelley's scaling and support his hypothesized diffusivities very well.

II This quantifies the relative contribution of heat and salt to the stratification--closer to 1 is more unstable (Turner. 1973).

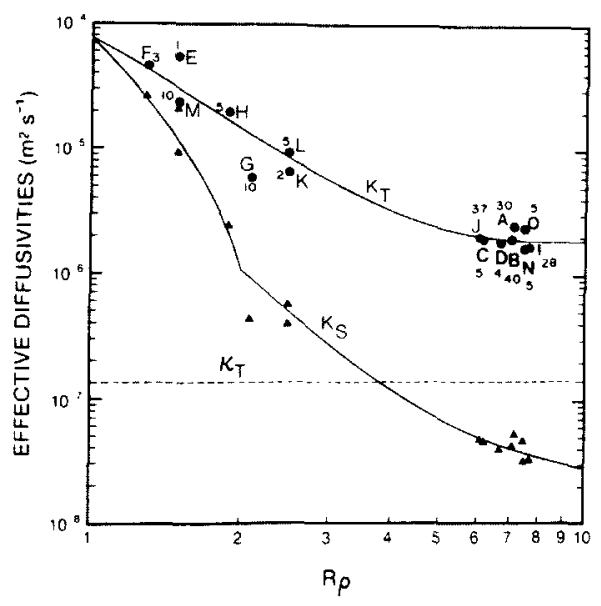

Fig. 4: Effective diffusivities for salt (triangles) and heat (circles) from Kelly (1984). The molecular diffusivity for heat is shown for reference. The letters represent data sources listed in Table 1 of Kelley (1984), and the numbers are 1,000 times the buoyancy frequency $\left(s^{\prime}\right)$. The solid curves are the proposed diffusivity formulations based on dimensional reasoning, laboratory flux measurements, and empirical observations of layer thicknesses. The curves to the left of data point $F$ are quite uncertain due to sparse laboratory measurements.

Note that Kelley's diffusivities for heat are greater than for salt. The effective diffusivity for density turns out to be negative due to the dominance of the (upward) heat flux.

Padman and Dillon's observations and analysis therefore support the laboratory "4/3" flux laws and Kelley's scaling hypothesis for layer thickness. for diffusive convection. The combination of the two results suggests that Kelley's diffusivity laws for diffusive sense thermohaline staircases may be generally valid-that diffusive fluxes have been quantified well enough to use in circulation models. However, it is important to recognize that these formulas can only hold where layers exist, and even then, that interactions with internal waves and turbulence may modify the fluxes.

\section{Salt Fingers-The C-SALT Experiment}

Northeast of Barbados, the Antarctic Intermediate Water flows underneath the warmer saltier subtropical underwater, making conditions right for strong salt fingering. Schmitt (1988) led a large experiment called C-SALT that aimed to quantify the fluxes. A CTD/AXBT (Air- launched eXpendable Bathy Thermograph) survey at the start of the experiment revealed that the stratification was broken into steps on the order of $20 \mathrm{~m}$ thick, with temperature contrasts between the steps on the order of 1 degree, and that the areal extent of the steps was one million square kilometers. The layers were laterally coherent, changing $\mathrm{T}$ and $\mathrm{S}$ along the layers in a manner consistent with the known salt finger flux ratio. Surprisingly. however, microstructure surveys of the region, both drops and tows, revealed turbulence dissipation levels an order of magnitude lower than were expected from extrapolation of the laboratory flux laws. This seems to be associated with the fact that observed interfaces were a few meters thick. whereas laboratory measurements (which always started with thin interfaces) led investigators to expect $20 \mathrm{~cm}$ thicknesses. In addition, individual interfaces were often upon closer inspection found to be broken into a series of several smaller interfaces. Kunze (1987. 1990, 1994) attributes the thickened interfaces to interactions with internal waves, shear, and perhaps turbulence. and has put forward theories to explain the lower fluxes in terms of the thickened interfaces. Despite this complication in extrapolating laboratory measurements to the ocean, the observations indicate very significant fluxes. Schmitt (1988) estimates the salt diffusivity to be $10^{-4} \mathrm{~m}^{2} / \mathrm{s}$. with a net salt flux comparable with that of the remainder of the N. Atlantic subtropical gyre. So, although it seems that salt fingers are dominant in the C-SALT area, more work is needed to understand why the laboratory measurements don't scale directly to the ocean.

\section{Effects on Thermohaline Circulation}

One of the important large-scale consequences of double-diffusion is its effect on the strength of thermohaline circulation. Gargett and Holloway (1992) performed a very simple and striking experiment with a model of ocean circulation. First, they ran the model with equal diffusivities for heat and salt (Fig. 5, bottom panels), and found that it exhibited the commonly observed pattern with $\sim 8$ Sverdrups of sinking motion near the northern boundary and a broader upwelling zone to the south. The halocline was fairly broad and distributed through the water column. They then made one change: they forced the salt diffusivity to be twice that for heat, as would be appro- 
$\psi($ Zonal section)

S (Zonal section)
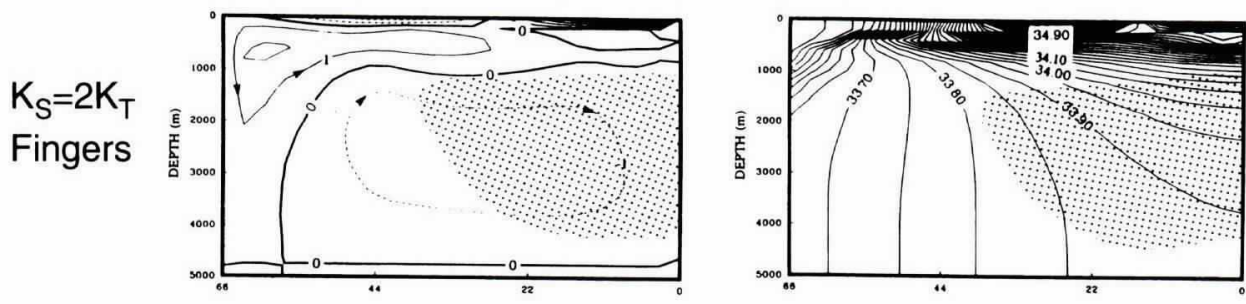

$\mathrm{K}_{\mathrm{S}}=\mathrm{K}_{\mathrm{T}}$ Control

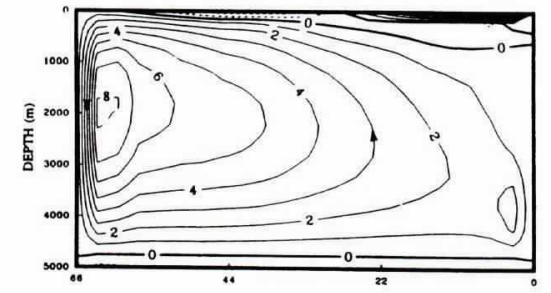

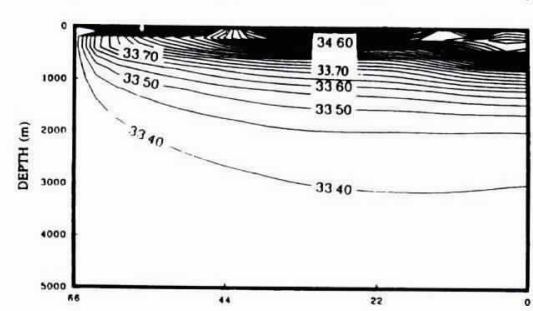

Fig. 5: The Meridional overturning streamfunction (left panels) and Salinity (right panels) for two experiments on an ocean circulation model, from Gargett and Holloway (1992). The bottom panels show a "control" experiment for which heat and salt diffusivities are equal, and the density diffusivity is therefore positive. The top two panels show an experiment for which the heat diffusivity is chosen to be twice the salt diffusivity. In the top two panels, the region of effectively negative density diffusivity is shown as a hatched region.

priate for salt fingers. ${ }^{\text {TI }}$ The overturning circulation was shut down, with a small weak cell of $\sim 2$ Sverdrups restricted to the northern corner of the domain. The circulation in the subtropical deep water was reversed, with weak sinking below $2,000 \mathrm{~m}$. The deep water had uniform salinity and the halocline was pushed up to within $500 \mathrm{~m}$ of the surface. The shaded region in Figure 5, top panels, corresponds to the region of upgradient density flux, where $\mathrm{K}_{\rho}$ is effectively negative. Note that it corresponds to the region of sinking, where the deep salinity is uniform. This is the reason for the shutdown of the thermohaline circulation-to understand this, we reexamine the classical thermocline balance.

Figure 6a shows the classical thermocline balance postulated by Munk (1966). The balance between upwelling of cold deep water, and the mixing downward of warmed surface waters allows a steady state to be achieved with a temperature structure of the form $\mathrm{T}(\mathrm{z}) \sim \exp \left(\mathrm{wz} / \mathrm{K}_{\mathrm{T}}\right)$, which describes a surface thermocline

\footnotetext{
"ll Gargett and Holloway (1992) also performed an experiment with the salt diffusivity half that for heat, as would be appropriate for diffusive stratification. However, very little of the water column was diffusively stratified, so that experiment was not very realistic.
}

boundary layer. Consider what happens if we demand this balance to hold for density, with a negative density diffusivity,
$\mathrm{K}_{\rho}$, that is expected to hold for salt fingers (Fig. 6b). First, the downward density flux will tend to make the surface waters lighter, which cannot balance the buoyancy flux into the surface. The advection/diffusion balance demands a density profile of the form $\rho(\mathrm{z}) \sim \exp \left(\mathrm{wz} / \mathrm{K}_{\rho}\right)$ and this looks like $\exp (-\mathrm{z})$ for negative density diffusivity, which decays upward. Thus the balance demands a bottom pycnocline, which is incompatible with the surface thermocline demanded by a positive $\mathrm{K}_{\mathrm{T}}$. This can be seen more simply by noting that it is dense water that upwells from the deep, and the downward density flux only tries to make this more dense, so a balance can't easily be achieved.

Negative density diffusivity has a more subtle effect through the vortex stretching dynamics of Stommel et al. (1958). If water is upwelling into the thermocline, water columns in the thermocline must be squashed, and this combines with the linearized vorticity equation

$$
\beta \mathrm{v}=\mathrm{fw}_{\mathrm{z}}
$$

where $\beta$ is the $\mathrm{N}-\mathrm{S}$ gradient of the Coriolis parameter, $\mathrm{f}$, and $\mathrm{v}$ the meridional velocity. Thus, in the northern hemisphere,

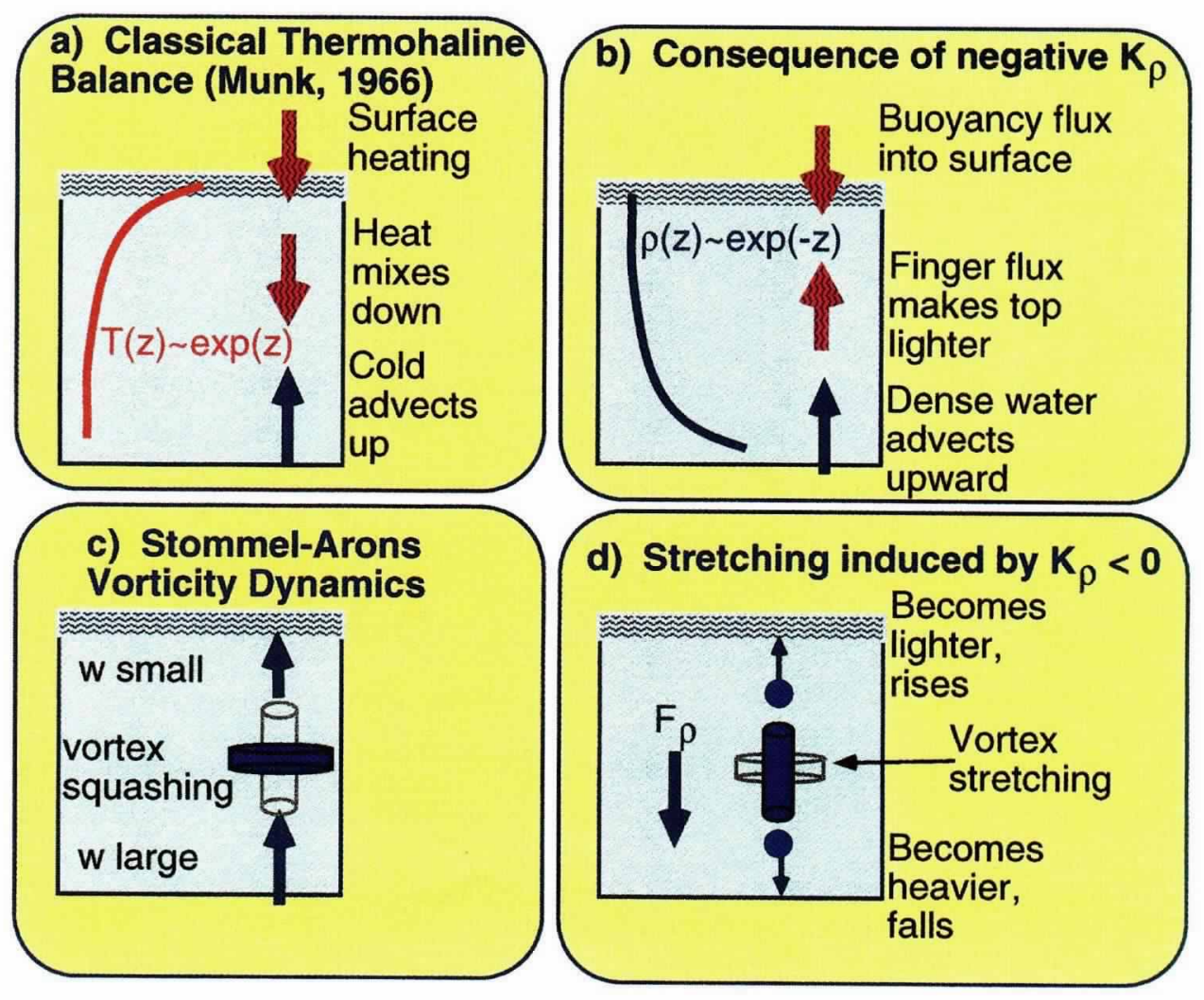

Fig. 6: Effect of negative density diffusivity on the classical thermocline balance, and on vortex stretching dynamics. 
where $\beta$ and $\mathrm{f}$ are positive, vortex squashing demands southward flow in the thermocline waters, and Northward flow in the deep waters.

Now consider the effect of a peak downward density flux at one particular level. Water above that level is made lighter and will rise, whereas water below that level becomes heavier and sinks. The vortex stretching in between demands northward flow through the vorticity equation. Thus a negative density diffusivity has the potential to reverse the vertical stretching effects, and therefore the meridional flows, of the thermohaline circulation.

The effects of differential diffusivities on thermohaline circulation have been examined in very simple models and experiments, involving rudimentary mixing parameterizations and model geometries (Gargett and Holloway, 1992), or simple extensions of the Stommel box model (Gargett and Ferron, 1996). There is much work to be done with more complex and precise parameterizations, and including more realistic coastlines, bathymetry, and model geometry, some of which is currently underway (J. Zhang and W. Merryfield, personal communication).

\section{Thermohaline Intrusions}

The most intriguing effect (to me, at least) of double-diffusive vertical fluxes is the growth of thermohaline intrusions. These are quasi-horizontal layering motions that advect water laterally across a frontal boundary and cause lateral mixing. They are best illustrated (Fig. 7) with a laboratory experiment (Ruddick and Turner, 1979); a video of this experiment can be seen in the physical oceanography lab demos section of the Dalhousie University Oceanography web page <http://www. phys.ocean.dal.ca/DalOcean_Home.html>.

In this experiment, a long tank is stratified with a vertical gradient of sugar solution on the left, and salt solution on the right, with equal densities at the same level on either side.\# When the barrier separating the two tank halves is removed and the small internal wave disturbances die out, a series of intrusive layers forms

\# To avoid problems of heat loss, these experiments used the analog sugar/salt system. Since the molecular diffusivity of sugar is about three times smaller than for salt, the sugar solution ("high-S") plays the role of oceanic warm, salty water, while the laboratory salt solution ("high-T") plays the role of oceanic cool, fresh water.

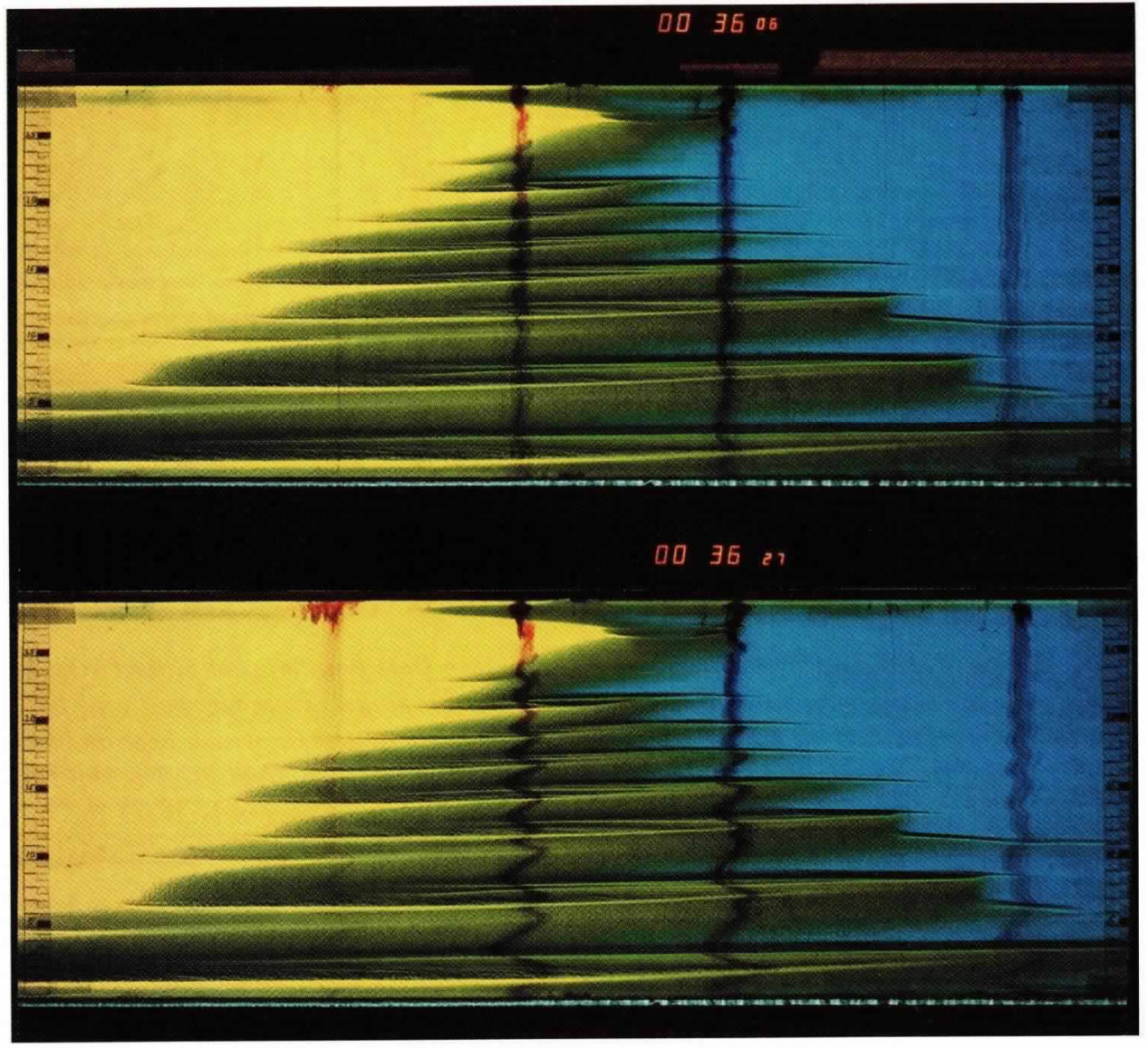

Fig. 7: The developed stage of a laboratory model of thermohaline intrusions. The time of each photograph (hh:mm:ss) is shown immediately above each photo, and the distortions of the purple dye streaks indicate the quasi-horizontal velocity field.

and grows laterally. The layers are thicker near the bottom, where the salt/ sugar contrast is larger, and the speed of advance of the layers is proportional to their thickness.

The layers are driven by the density changes that double-diffusive fluxes create. The feedback cycle is the following (Fig. 8):

1. Lateral advection in alternating directions draws high-S solution (in the ocean this would be warm, salty water) above high-T solution (in the ocean this would be cool fresher water), creating a vertical stratification suitable for "sugar" fingers.

2. The vertical density flux of the "sugar" fingers causes the high-S layers to become less dense, and the high-T layers to become more dense, as each layer continues to advect.

3. The changes in density in step 2 above cause the high-S layers to rise as they advect to the right, and the high-T layers to fall as they advect to the left. This creates the systematic tilt from lower left to upper right.
4. The slight density anomaly of each layer, combined with the tilt, creates pressure perturbations that drive the advective motions in step 1 .

Of course, between each finger region is a diffusive region (sugar solution below salt solution), and if the density flux in the diffusive regions were to dominate, the layers would slope the opposite way. This was observed in the laboratory experiments in some instances, and also occurs in the ocean.

How do salt fingers drive lateral interleaving?

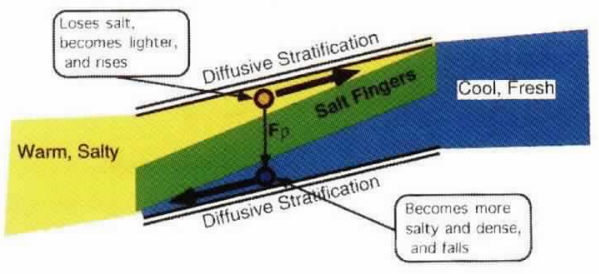

Fig. 8: Links between finger buoyancy flux and intrusion slope. 
Intrusions are extremely common and have been found in coastal shelf break fronts (Horne, 1978), frontal boundaries between water masses like the Antarctic Circumpolar front (Joyce et al., 1978) and in eddies of anomalous water such as Meddies. In the case of Meddy Sharon (Ruddick and Hebert, 1988), intrusions were found to be the single most significant mixing agent, causing the anomaly to be lost on a time scale of a year. Furthermore, the Meddy intrusions were found by their slope and density anomaly to be driven by double-diffusive vertical fluxes (Ruddick, 1992; Ruddick and Walsh, 1995). Intrusions crossing the equatorial Atlantic have been found to be coherent over scales of hundreds of kilometers (Richards and Pollard, 1991 ).

The most spectacular intrusions discovered to date are in the Arctic Ocean, described by Carmack et al. (1995). These have $\mathrm{T}-\mathrm{S}$ properties that can be tracked coherently over a lateral extent of several thousand kilometers. The intrusions become more dense as one moves away from the warm, salty side, and systematically exhibit lower than average density gradient in the salt finger regions. These observations are consistent with the intrusions being dominated by the diffusive fluxes rather than finger fluxes. However, May and Kelley (1997) show that a thermohaline front with baroclinic shear can be unstable to a new class of interleaving that uses the potential energy of the baroclinicity. The Arctic intrusions could be driven at least partially by baroclinicity while they are growing, but they must switch over to the diffusively-dominated flux state in order to achieve a steady advective-diffusive balance. On the basis of the temporal changes found over the last decade, Carmack et al. (1995) suggest that these intrusions may be carrying signals of global change into the Arctic Ocean.

\section{Summary}

The unique and nonintuitive feature of double-diffusion is downward density flux, which constitutes a negative eddy diffusivity for density. This provides the potential energy that drives the mixing and is an important part of the mechanism that creates layers. Layer formation can enhance double-diffusive fluxes, although interactions with shear, internal waves. and turbulence may change the fluxes considerably from those measured in the laboratory. The upgradient density flux has consequences for the larger scale. Thermohaline circulation can be shut down because the classical vertical upwelling-diffusion balance is severely upset having a negative density diffusivity. On an intermediate scale, lateral T-S gradients combine with vertical doublediffusion to form thermohaline intrusive layers, in which lateral mixing over scales up to thousands of kilometers is driven by the density changes induced by double-diffusion.

\section{Acknowledgements}

I thank Ann Gargett for giving me the opportunity to describe this topic to many who might not otherwise learn of it. I'm grateful to my colleagues Dan Kelley, Brian May, Eric Kunze, and Ann Gargett for their comments on the manuscript. My work is supported by the Natural Sciences and Engineering Research Council of Canada.

\section{References}

Carmack, E.C., K. Aagaard. J.H. Swift. R.G. Perkin. F.A. McLaughlin. R.W. Macdonald and E.P. Jones, 1998: Thermohaline transitions. In: Physical Processes in Lakes and Oceans. J. Imberger, ed. International Union of Theoretical and Applied Mechanics.

Gargett. A.E., 1988: The scaling of turbulence in the presence of stable stratification. $J . G e()$ phys. Res., 93, 5021-5036

and G. Holloway. 1992: Sensitivity of the GFDL ocean model to different diffusivities for heat and salt. J. Phys. Oceanogr., 22, $1158-1177$

and B. Ferron, 1996: The effects of differential vertical diffusion of $T$ and $S$ in a box model of thermohaline circulation. J. Mar: Res.. 54. 827-866.

Horne. E.P.W., 1978: Interleaving at the subsurface front in the slope water off Nova Scotia. $J$. Geophys. Res., 83. 3659-3671.

Huppert, H.E. and Linden, P.F.. 1979: On heating a stable salinity gradient from below. I. Fluid Mech., 95, 43I-464

Joyce. T.M.. W. Zenk and J.M. Toole. 1978: The anatomy of the Antarctic Polar Front in the Drake Passage. J. Geop/ys. Res., 83, 6(1)93. 6113

Kelley, D.E.. 1984: Effective diffusivities withm oceanic thermohaline staircases. J. Geophss. Res., 89, 10,484-10.488.

Kunze. E.. 1987: Limits on growing, tinite length salt fingers: A Richardson number constraint. J. Mar. Res., 45, 533-556.

. 1990: The evolution of salt fingers in inertial wave shear. $J$. Mar. Re's. $48,471-504$.

1994: A proposed flux constraint for salt fingers in shear. J. Mar. Re's. 52, 9991016

May, B.D. and D.E. Kelley, 1997: Effect of haroclinity on double-diffusive interlearing. $J$ Phys. Oceanegr.. 27, 1997-2008.

Munk, W.H., 1966: Abyssal recipes. Deep-Sea Res., 13. 707-730

Padman. L. and Dillon. T.. 1987: Vertical heat fluxes through the Beaufort Sea thermohaline staircase. $J$. Geophys. Re's. 92, 10.799 10,806

and Dillon. T., 1989: Thermal microstructure and internal waves in the Canada Basin diffusive staircase. Deep-Sea Res.. 36, 531$54 ?$

Richards, K.J. and R.T. Pollard. 1991: Structure of the upper ocean in the western equatorial Pacific. Nature, 350, 48-50)

Ruddick. B. and D. Hebert. 1988: The mixing of Meddy "Sharon". In: Simall-Scale Miving $m$ the Ocean. Elscvier Oceanography Serles. rol. 45. J.C.J. Nihoul and B.M. Jamurt. eds. Elsevier. New York. $541 \mathrm{pp}$.

and J.S. Turner, 1979: The vertical length scale of double-diffusive intrusions. DecopSea Res., 26A, 903-913.

and D. Walsh, 1995: Observations of the Density Perturbations which drive Thermohaline Intrusions. In: Double-Diffusive Comrection. A. Brandt and H.J.S. Fernando, eds Am. Geophysical Union. Washington. D.C.. $334 \mathrm{pp}$.

1992: Intrusive mixing in at Mediterranean salt lens-intrusion slopes and dynamical mechanisms. J. Phws. Oceonogr., 22. 12741285.

Schmitt, R.W., 1988: Mixing in a thermohaline staircase. In: Small-Scale Miximg in the Ocean, Elsevier Oceanography Series, vol. 46. J.C.J. Nihoul and B.M. Jamart. eds. El sevier, New York, $541 \mathrm{pp}$.

Shirtcliffe. T.G.L.. 1967: Thermonolutal convecton: observation of an overstable mode. Nature. 213, $489-490$

Stommel, H.. A.B. Arons and A.J. Faller. 1958: Some examples of stationary planetary flow patterns in bounded basins. Te'llus. 10, 179187.

Turner, J.S., 1973: Buswancy Effects in Fluids (Chapter 8). Cambridge University Press. Cambridge, U.K. 368 pp. コ 